\title{
CLODRONATE FOR TREATMENT OF BONE METASTASES IN HORMONE REFRACTORY PROSTATE CANCER
}

\author{
FLAVIO HERING, PAULO R.T. RODRIGUES, MARCO LIPAY \\ Portuguese Beneficence Hospital and Santa Helena Hospital, São Paulo, SP, Brazil
}

\begin{abstract}
Introduction: Approximately $85 \%$ of patients who die from prostate cancer have bone metastases. Even though the radiological aspect of such metastases is osteoblastic, we currently know that these lesions are mixed, with coexisting blastic and lytic lesions, always beginning with bone lysis by osteoclast proliferation. Treatment options are palliative and have poor response, and when there is an improvement it is usually short-lived. This work intends to study the effect of clodronate in the treatment of skeletal complications of prostate cancer.

Materials and Methods: In an open prospective study 32 patients with hormone refractory prostate cancer with metastases to bones were assessed, in the period between November 2000 and September 2002. Mean age was 69 years ( 51 to 83 years). Patients were previously assessed by a pain scale and Karnofsky index. They underwent bone scintigraphy, X-ray, dosage of prostate specific antigen (PSA) and biochemical tests before and following treatment (administration of intravenous clodronate every 28 days). The Student's t-test was used for statistical analysis.

Results: Twenty-nine patients (90.6\%) showed improvement after the first and the 2nd cycles, which persisted for at least 4 months. Average on the pain scale improved from 7.7 to 2.1 and Karnofsky index raised from 42 to 71 . Radiological aspect of the metastases improved in 15 patients $(46.8 \%)$ and side effects were low (only 2 patients $-6.2 \%$ ).

Conclusion: Bisphosphonate was effective in the treatment of skeletal complications of prostate cancer, presenting an objective response in $90.6 \%$ of treated patients, with a marked improvement in the pain scale, Karnofsky index and consequently in the quality of life of patients, and with low side effects.
\end{abstract}

Key words: prostatic neoplasms; neoplasm metastasis; bone; bisphosphonates; palliative treatment Int Braz J Urol. 2003; 29: 228-233

\section{INTRODUCTION}

The incidence of prostate adenocarcinoma has increased greatly in the last decade and, currently it is the most common type of non-cutaneous cancer found in men over 50 years old. During the year of 2002, approximately 210,000 new cases were diagnosed in the USA. Currently, at the time of diagnosis, even with all efforts for an early detection, $20 \%$ of patients present bone metastases already. Considering that about $30 \%$ of patients who receive treatment for localized prostate cancer presented a relapse, the number of patients with advanced disease is high. Most of such patients who are under hormone treatment become resistant to the hormone between 18 and 36 months after the therapy initiation and $85 \%$ to 95\% of these patients will present bone metastases and their complications (such as pain, fractures and 
plegias) (1-3). The patient who presents already bone metastases has an average survival of 2 years, which is a very long time, considering that he will suffer with skeletal complications (2-4).

The majority of bone metastases of prostate cancer are visualized on plain radiography as osteoblastic lesions, however they always begin as lytic lesions with osteolysis caused by the tumorous prostatic cells that interrupt normal bone remodeling through irregular stimulation of osteoclasts and osteoblasts. Following the lytic lesion, there is an excessive deposit of lamellar bone in the lacunae, forming the osteoblastic aspect. This mechanism results in osteosclerosis, increasing bone volume and destroying the normal bone (5-7).

Bisphosphonates have been widely employed in bone metastases caused by breast cancer and multiple myeloma with excellent results. It is a potent inhibitor of bone reabsorption, both normal and pathologic, exerting a depressant effect on mature osteoclasts and inhibiting the stimulation of osteoblasts, thus decreasing skeletal complications (6).

The objective of the present study is to evaluate clinically the effect of bisphosphonate clodronate in patients with bone metastases due to hormone refractory prostate adenocarcinoma.

\section{MATERIALS AND METHODS}

Between November 2000 and September 2002, 32 patients with bone pain due to metastasis from hormone refractory prostate adenocarcinoma were evaluated, and treated with clodronate in an open prospective study.

Mean age was 69 years (51 to 83). Among those, 9 had undergone orchiectomy, 11 were using cyproterone acetate, 6 used LH-RH analogs, 2 used bicalutamide, and 4 patients were using estrogens. $\mathrm{Pa}$ tients were considered hormone-refractory after 3 consecutive increases of PSA during hormone therapy. In 8 patients mitoxantrone and corticoids were administered, 5 patients received estramustine phosphate, 4 patients used ketoconazole with corticoids and 3 patients used PC-SPES. These medications were suspended when clinical or biochemical response ceased.
Before administration of first clodronate dose, patients underwent a skeletal mapping for recording the quantity and location of metastases, and every point with hypercaptation highly suggestive of metastases was radiographed.

All patients answered to a visual pain scale from 0 to 10 (no pain, to very strong pain), and general conditions were assessed by Karnofsky index. Analgesics were taken as necessary, and these patients were contacted every 14 days (office visits or telephone calls). Those who showed no improvement after the second cycle received adjuvant treatments (radiotherapy, chemotherapy or samarium).

After 4 months of treatment the patients underwent a new bone scintigraphy for comparison with the initial one. Radiographs of metastases locations were also taken. Monthly, determination of PSA, phosphatases, calcium, creatinine, transaminases, and hemogram with erythrocyte sedimentation rate were made.

Clodronate was administered intravenously every 28 days at a dosage of $1,500 \mathrm{mg}$ ( 5 vials of 300 $\mathrm{mg}$ diluted in $500 \mathrm{ml}$ of saline solution, infused in $2 \mathrm{~h}$ ). The procedure was done at an outpatient basis without requiring admission to hospital. Following the infusion, the patient was released for his normal activities.

For parametric and non-parametric statistical analysis of variations in the pain scale and in the Karnofsky index, the Student's t-test was used for comparing data before and during treatment.

\section{RESULTS}

According to the visual pain scale, 29 patients (90.6\%) showed improvement, with 27 (84.3\%) having a score equal or superior to 5 (Table-1).

A total of 29 patients $(90.6 \%)$, responded to treatment with bisphosphonate. Radiologically, an improvement in metastases was observed after the first 6 months in 15 patients $(46.8 \%)$, Table- 2 .

The average value on the visual pain scale before treatment was 7.7, and after treatment it decreased to 2.1. Of the 32 treated patients, $29(90.63 \%)$ responded to treatment with bisphosphonate and the Karnofsky index improved from 42 (range 32 to 58) to 71 (range 50 to 82 ), Table-3. 
Table 1 - Improvement in pain according to visual scale after 2 cycles of bisphosphonate.

\begin{tabular}{lrc}
\hline $\begin{array}{l}\text { Pain Scale Level } \\
\text { Following Clodronate }\end{array}$ & Patients & $\%$ \\
\hline$<5$ & 27 & 84.38 \\
$\geq 5$ & 2 & 6.25 \\
Without improvement & 3 & 9.37 \\
Total with improvement & 29 & 90.63 \\
\hline
\end{tabular}

Table 2 - Improvement in radiological image after treatment with bisphosphonate.

\begin{tabular}{lrl}
\hline Type & $\mathbf{N}^{\mathbf{0}}$ & $\boldsymbol{\%}$ \\
\hline Osteoblastic & 12 & 48 \\
Osteolytic & 3 & 42.8 \\
Total & 15 & 46.8 \\
\hline
\end{tabular}

Side effects were minimal, with findings of epigastralgia and excessive salivation in 1 patient, and superficial phlebitis in left upper limb in another (Table-4).

\section{DISCUSSION}

It is known those bone metastases are the most frequent ones in advanced prostate cancer. It is known that approximately $85 \%$ of patients who die with prostate adenocarcinoma have skeletal lesions and complications such as pain, pathological fractures or spinal compression (4).
Based on clinical and experimental studies, one can conclude that bone metastasis both in prostate and in breast cancer have a similar behavior, and in the latter bisphosphonates are widely employed as an adjuvant treatment with excellent results (7). Recently a double-blind study was published, involving 1,069 patients with breast cancer where it was concluded that the use of bisphosphonate clodronate had a significant influence on the increase of survival (8).

Recent researches demonstrated that in the physiology of bone metastasis, either in multiple myeloma, or in prostate or breast cancer, the key to the lesion's initiation is an increase in osteoclastic activity promoting a bone lysis. Subsequently an excessive and disordered deposit of sclerotic bone will occur due to the increase in osteoblasts activity (osteoblastosis). In the case of prostate cancer, such deposit is what most often confers the radiological aspect of an osteoblastic lesion (6).

Cancerous prostatic cell detach from prostate, reach blood circulation and are attracted to bone by local and distant chemotactic factors such as metalloproteinases. Among the main local factors are growth factors and cell-cell and cell-matrix interactions, favoring thus the installation and growth of metastasis in the bone (6,9-12). The release of paracrine factors that promote the initial bone absorption is triggered by osteoclasts activation, initiating the lytic lesion. The most important factors are: interleukin 1, 6 and 11; $\alpha$ and $\beta$ tumor necrosis factor, epidermal growth factor, parathormone-related peptide, prostaglandins and procalepsin D. Factors that promote the increase of osteoblastic activity are released subsequently, which will allow the excessive bone deposit over the initial lytic lesion. Among the most important are the fibroblastic growth factor,

Table 3 - Summary of patients who underwent treatment with bisphosphonate.

\begin{tabular}{lccc}
\hline Parameters & Patients & Before Treatment & After Treatment \\
\hline Average of pain scale score (0-10) & 32 & 7.7 & 2.1 \\
No. of patients who had a response & 3 & - & 29 \\
Average of Karnofsky index & 32 & $42(32 \%$ a 58\%) & $71(50 \%$ a $82 \%)$ \\
\hline
\end{tabular}


Table 4 - Side effects.

\begin{tabular}{lcc}
\hline Type & Patients & $\%$ \\
\hline Slight digestive disorders & 1 & 3.1 \\
Superficial phlebitis & 1 & 3.1 \\
Total & 2 & 6.2 \\
\hline
\end{tabular}

bone morphogenetic protein and insulin-like growth factor $(6,9-11,13)$.

Bisphosphonates cause a decrease in osteoclasts activity, inhibiting bone lysis, and act also on osteoblasts preventing the hyperactivity of these cells (osteoblastosis) that culminates with excessive bone formation. A direct action over the tumor was also described, inhibiting angiogenesis and promoting the apoptosis of cancerous cells, in addition to inhibiting metalloproteases and cytokines (1,7-9,13).

According to their molecular mechanism of action, bisphosphonates are divided in 2 large groups: amino bisphosphonates (NBPs) and non-amino bisphosphonates (non-NBPs). Differently from nonNBPs (clodronate and ethidronate), NBPs (pamidronate, ibandronate, alendronate and zolendronate) do not have a nitrogen atom in their formulation, what confers a different mechanism of action as for the molecular aspect. While NBPs act intracellularly, inhibiting the mevalonate cycle, nonNBPs act by promoting a direct toxicity over osteoclasts and osteoblasts inducing the apoptosis and inhibiting the ATP/ADP translocase enzyme $(9,15,16)$. The differences between the 2 groups' mechanism of action determine differences in their effects, such as for instance, their activity over the inflammatory process. Non-NBPs such as clodronate have anti-inflammatory action and anti-rheumatic activity that occur with NBPs (16).

Pre-clinical studies demonstrate that osteolytic lesions always precede the abnormal bone deposit (osteoblastosis), what confer the osteoblastic aspect to the majority of metastatic skeletal lesions of prostate cancer (6). It was shown through biopsies of metastatic skeletal lesions that excessive bone reabsorption occurs at these sites, similarly to breast cancer $(6,7,13)$. The rational for using bisphosphonates in prostate cancer comes from studies that demonstrated their beneficial effects in inhibiting the invasion of prostate cancerous cells as well as their adherence and lysis of bone matrix. Another advantage of clodronate over other bisphosphonates is the possibility of using it orally, since its absorption and effects have been already demonstrated in clinical use (13).

As we have seen, bisphosphonates are used in breast cancer and multiple myeloma since the early 80s with excellent results, however only in the beginning of the $90 \mathrm{~s}$ the first studies with bisphosphonates in prostate cancer have appeared $(9,15)$. The first study with bisphosphonate in prostate cancer was conducted with clodronate by Adami et al. in 1985 (17), who treated 17 patients with osteoblastic metastasis by administering $300 \mathrm{mg}$ of daily intravenous clodronate during 14 days and 3,200 mg orally during the other 4 to 11 weeks. Significant improvement was observed in pain and in Karnofsky index in 16 patients $(94.1 \%)$ for 4 to 8 weeks.

In another study, Verreuther (13) administered to 41 patients with skeletal lesions due to prostate cancer, intravenous clodronate in a dosage of $300 \mathrm{mg}$ daily for 8 days, along with 1,600 mg orally. Twenty patients $(71 \%)$ presented a significant improvement in pain as soon as in the first 3 to 5 days and 9 remained asymptomatic.

Heidenreich et al. (2001) treated 85 patients with skeletal complications due to metastatic prostate cancer with $300 \mathrm{mg}$ of daily intravenous clodronate for 8 days, and 1,600 mg daily by oral route for maintenance. They presented a response with an important decrease in pain in 64 patients $(75 \%)$ and $19(22 \%)$ became asymptomatic, with improvement in Karnofsky index from $45 \%$ to $70 \%$ (18).

These studies corroborate our results. As well as in the paper by Adami et al. (17) more than $90 \%$ of our patients had a significant improvement in pain, with 27 of 32 patients staying below $5(0-10)$ in the pain visual scale score and improvement in Karnofsky index from $42 \%$ (range $32 \%$ to $55 \%$ ) to $71 \%$ (range $50 \%$ to $82 \%$ ), following treatment with clodronate. In our study we used intravenous clodronate in bolus, at the posology of $1,500 \mathrm{mg}$ (5 vials containing 
$300 \mathrm{mg}$ ) diluted in $500 \mathrm{ml}$ of saline solution applied during 2 h every 28 days, and performed at an outpatient basis.

Side effects were observed in only $6.2 \%$ of cases, and those were diarrhea and epigastralgia in one patient and superficial phlebitis in another, what is close to Verreuther's results (13) and is lower than the complication reported by Heidenreich et al. that is $17 \%$ (18). Maybe this fact has occurred due to the association with oral clodronate used by these authors for maintenance of treatment and that can lead to slight digestive disorders.

Following administration, 70 to $80 \%$ of the active substance is cleared by urine in up to $24 \mathrm{~h}$. Clodronate's advantage relative to other bisphosphonates is the possibility of using it orally. Smith (19) used oral ethidronate and did not observe patients' improvement in relation to the placebo group, because this medication had a very low absorption by this route, becoming ineffective and failing to produce the desired effect.

Among our patients, 25 (78.12\%) had osteoblastic and 7 (21,8\%) osteolytic bone metastasis. Both types responded well to treatment with clodronate. Pelvic bones were the most affected by single metastasis, and associated lesions were the predominant (50\%). In 15 patients $(46.8 \%)$ there was improvement and even disappearance of lesions in radiological studies. This effect can be explained by the direct action that clodronate exerts over the metastatic tumorous cell (induction of apoptosis, inhibition of angiogenesis and cytostatic effect) $(8,9,14)$.

In conclusion, one can verify that clodronate is an effective drug for palliative treatment of bone complications caused by hormone refractory prostate cancer, with prompt effects on pain, few side effects and a significant improvement in the quality of life.

\section{REFERENCES}

1. Berruti A, Dogliotti L, Bitossi R, Fasolis G, Gorzegno $\mathrm{G}$, Bellina $\mathrm{M}$, et al:: Incidence of skeletal complications in pattents with bone metastatic prostate cancer and hormone refractory disease: predictive role of bone resorption and formation markers evaluated at baseline. J Urol. 2000; 164: 1248-53.
2. Greenlee RT, Murray T, Bolden S: Cancer statistics, 2000. American Cancer Society 2000; 50: 6-11.

3. Parker SL, Tong T, Bolden S: Cancer statistics 1997. Ca Cancer J Clin. 1997; 47: 5-6.

4. Issacs JT: The biology of hormone refractory prostate cancer. Why does it develop? Urol Clin North Am. 1999; 26: 263-9.

5. Smith JA Jr., Soloway MS, Young MJ: Complications of advanced prostate cancer. Urology 1999; 54: 8-12.

6. Dodwell DJ: Malignant bone resosption: Cellular and biochemical mechanisms. Ann Oncol. 1992; 3: 25767.

7. Guise TA, Mundy GR: Cancer and bone. Endocr Rev. 1998; 19:18-54.

8. Diel IJ, Solomayer EF, Costa SD: Reduction in new metastase in breast cancer with adjuvant clodronate treatment. New Engl J Med. 1998; 339:357-63.

9. Powles T, Paterson S, Kants JA, McCloskey E, Ashley S, P-Ilkkanen: Controled trial of clodronate in patient with primary operable breast cancer. J Clin Oncol. 2002; 20: 3219-24.

10. Orr F, Lee J, Duivenvoorden WC: Pathophysiologic interactions in skeletal metastasis. Cancer 2000;88 (Suppl 12): 2912-5.

11. Sansoni P, Passeri G, Fangnoni F, Mohagheghpour N, Snelli G, Brianti V et al.: Inhibition of antigen-presenting cell function by alendronato in vitro. $\mathrm{J}$ Bone Miner Res. 1995; 10: 1719-23.

12. Lissoni P, Cazzanigna M, Barni, Derenne S: Acute effect of pamidronato administratton on serum levels of interleukin- 6 in advanced solid tumor patients with bone metastase and their possible implications in the immunoterapy of cancer with interleukin-2. Eur J Cancer 1992; 33: 304-8.

13. Verreuther R: Bisphosphonates as an adjunct to palliative therapy of bone metastases from prostatic carcinoma. A pilot study on clodronate. Br J Urol. 1993; 72: 792-5.

14. Nakashima J, Tachibana M, Horiguchi Y: Serum Interlevkin-6 as a prognostic factor in pacients with prostate cancer. Clin Cancer Res. 2000; 6: 2702-5.

15. Munoy GR: Bisphosphosnates and tumor burden. J Clin Oncol. 2002; 20: 3191-2.

16. Luckman SP, Huges DE, Loxon FF: Nitrogen containing bisphophonates inhibit the mevalonate pathway and prevent post-translational prenylation of GTP-binoing proteins including. Ras J Bone Miner Res. 1998; 13: 581-9.

17. Adami S, Salvagno G, Guarrera G: Dichloromethylene - bisphosphonate in patients with prostatic carcinoma 
metastatic to the skeleton. J Urol. 1985; 134: 1152-5.

18. Heidenreich A, Hofmann R, Engelmann UH: The use of bisphosphonate for the palliative treatment of painful bone metastasis due to hormone refractory prostate cancer. J Urol. 2001; 165: 136-40.
19. Smith JA Jr: Palliation of painful bone metastases from prostate cancer using sodium etioronate: Results of randomized, prospective, double-blind placebo controlled study. J Urol. 1989; 141: 85-7.

Received: January 14, 2003

Accepted after revision: April 11, 2003

\footnotetext{
Correspondence address:

Dr. Flávio Hering

Rua Teodoro Sampaio, 744 / 84

São Paulo, SP, 05406-000, Brazil

Fax: + 5511 3064-2989
} 